\title{
EFFECTS OF CLOSURE MODELS ON SUPERCAVITATING BUBBLE DEVELOPMENT
}

Bin-Chen Wu

Department of Systems Engineering and Naval Architecture National Taiwan Ocean University Keelung, Taiwan, R.O.C

Jiahn-Horng Chen

Department of Systems Engineering and Naval Architecture National Taiwan Ocean University Keelung, Taiwan, R.O.C, b0105@mail.ntou.edu.tw

Follow this and additional works at: https://jmstt.ntou.edu.tw/journal

Part of the Engineering Commons

\section{Recommended Citation}

Wu, Bin-Chen and Chen, Jiahn-Horng (2005) "EFFECTS OF CLOSURE MODELS ON SUPERCAVITATING BUBBLE DEVELOPMENT," Journal of Marine Science and Technology. Vol. 13: Iss. 3, Article 1.

DOI: $10.51400 / 2709-6998.2122$

Available at: https://jmstt.ntou.edu.tw/journal/vol13/iss3/1

This Research Article is brought to you for free and open access by Journal of Marine Science and Technology. It has been accepted for inclusion in Journal of Marine Science and Technology by an authorized editor of Journal of Marine Science and Technology. 


\section{EFFECTS OF CLOSURE MODELS ON SUPERCAVITATING BUBBLE DEVELOPMENT}

\section{Acknowledgements}

This work was supported by the National Science Council, Republic of China, under the grant NSC912815-C-019-013-E. The authors would like to express their thanks to this support. 


\title{
EFFECTS OF CLOSURE MODELS ON SUPERCAVITATING BUBBLE DEVELOPMENT
}

\author{
Bin-Chen $\mathrm{Wu}^{*}$ and Jiahn-Horng Chen*
}

Key words: 2-D supercavitation, closure model, hydrofoil.

\begin{abstract}
Effects of different cavity closure models on the development sheet supercavitation of two-dimensional hydrofoils were examined in this report. The study was carried out under the assumption of potential flow for which a cavity closure model was needed. A potential-based boundary element method has been developed for this purpose. The models we employed included the constant velocity model and the short plate termination model. Several different airfoil shapes were tested. Their effects on the cavity shape, lift coefficient, cavity volume and computation efficiency were studied. Careful investigations show that closure models have insignificant influence on physical quantities, but do have strong effects on computational efficiency.
\end{abstract}

\section{INTRODUCTION}

Cavitation is a challenging hydrodynamic phenomenon both in physical science and engineering applications. In physical science, cavitating flows involve many complicated and interdisciplinary physical phenomena in which both physicists and applied mathematicians are interested. In engineering applications, erosion and corrosion of structures are almost unavoidable wherever cavitation occurs. Detrimental noise ensues from collapse of cavitating bubbles. More recently, new generation of underwater weapons has been developed by the concept of supercavitation.

In the past two decades, there has been significant progress in the study and observation of sheet cavitation phenomena, primarily due to the fast development of high-speed computation science and more advanced experimental techniques. Nevertheless, the investigation of cavitation modeling has a very long history that can be traced back to Kirchhoff [8]. Major pioneering development was theoretical [5,9]. The successful

Paper Submitted 06/30/04, Accepted 03/16/05. Author for Correspondence: Jiahn-Horng Chen.E-mail:b0105@mail.ntou.edu.tw.

*Department of Systems Engineering and Naval Architecture National Taiwan Ocean University Keelung, Taiwan, R.O.C. application of potential flow theory played an active role in these early theoretical developments. Literature surveys indicate that there are several significant features worth of attention. The cavity was usually macroscopically treated as a single big bubble of finite length, which encompasses the whole region where cavitations occur and micro bubbles dominate. In addition, the pressure inside the cavity bubble was usually assumed to be constant. Linearized theories were proposed by Tulin [13, 14]. He obtained the analytical solution for a supercavitating flat plate hydrofoil with a sharp leading edge. Later, Acosta [1] provided the first partial cavitation solution for a flat-plate hydrofoil. More recent linearized theoretical development was due to Furuya and Acosta [6]. Nonlinear development has been explored by $\mathrm{Wu}[18,19]$. He employed the openwake model to study the cavitating flat plate at an arbitrary angle of flow incidence.

However, in the context of potential flow, the specification of condition in the cavity closure region creates serious problems in cavitation modeling. It is clear that very complicated flow phenomena occur in this region and cannot be incorporated into a potential flow model. In fact, the termination of cavity has not yet been clearly described due to its turbulent, two-phase, and even compressible flow physics. Therefore, it is necessary to resort to some artifact in the vicinity in this region to cope with this difficulty. Several closure models have been devised (see, e.g. [2]). The models investigated in the present study will be addressed and discussed later.

With the modern evolution of computational methods, several nonlinear numerical procedures of boundary-element type have been successfully developed for the solution of sheet cavitation, based on the early theoretical achievements. In these approaches, cavity surface conditions are usually satisfied on the exact cavity surface that is part of the solution and determined iteratively by proper computational algorithms. Uhlman [15, 16] employed a velocitybased nonlinear boundary element method to obtain solutions for partially-cavitating and supercavitating 
hydrofoil flows. Kinnas and Fine [7] developed a potential-based nonlinear boundary element method. This appears to be superior to the velocity-based methods in terms of convergence. Several different cavity closure models have been incorporated in these computation procedures. For example, in partially cavitating flow computations, Kinnas and Fine [7] employed a model in which the velocity in the termination zone was decreased to zero according to a prescribed law. More recently, the reentrant jet was used by Krishnaswamy et al. [10]. Vaz et al. [17] developed a new boundary element method in which the elements are located on the foil surface and the boundary conditions for the cavity surface have been reformulated based upon a Taylor expansion. They compared their results with those using the reentrant jet model [4] and concluded that different models had insignificant effects on the cavity physics.

In supercavitating flow computations, Uhlman [16] employed the short-plate model; Lee et al. [11] and Chen and Weng [3] used a simple closure model. However, their effects on computations, such as convergence and convergence rate, and on flow physics, such as lift, cavity shape, and so on, have not yet been studied and compared in detail. It is the purpose of the present study to carry out a more clear observation in computation.

\section{THEORETICAL FORMULATION}

Consider a uniform potential flow past a twodimensional hydrofoil, as shown in Figure 1. The governing equation of the velocity potential $\phi=\phi(\mathbf{x})$ is the Laplace equation,

$$
\nabla^{2} \phi=0
$$

The flow velocity is related to the velocity potential by

$$
\mathbf{u}=\nabla \phi
$$

Several boundary conditions are identified for wellposedness of the problem.

For a uniform incoming flow, the velocity distribution far away from the hydrofoil is

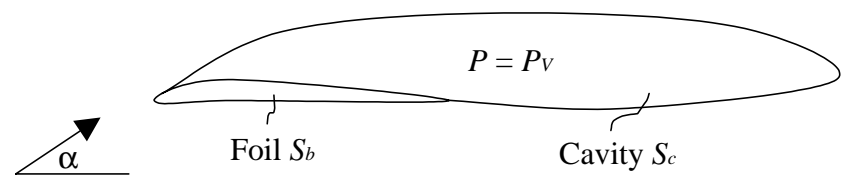

Fig. 1. Schematic of cavitating flow.

$$
\nabla \phi=U(\mathbf{i} \cos \alpha+\mathbf{j} \sin \alpha)
$$

where $\alpha$ is the angle of attack of the uniform flow, $U$ the speed of the incoming uniform flow, and $\mathbf{i}$ and $\mathbf{j}$ the unit vectors in $x$ - and $y$-directions, respectively.

On the surface of hydrofoil, $S_{b}$, and the surface of cavity, $S_{c}$, a kinematic condition must be prescribed,

$$
\nabla \phi \cdot \mathbf{n}=0
$$

where $\mathbf{n}$ denotes the unit normal to the surfaces. This condition guarantees no penetration of the fluid flow through the surfaces.

In addition, a dynamic condition is required on the surface of the cavity, $S_{c}$. That is, the pressure inside the cavity is constant,

$$
p=p_{v}=\text { constant }
$$

where $p_{v}$ denotes the vapor pressure. According to this condition, it can be derived via the Bernoulli equation that on the cavity surface, we have

$$
\left|\mathbf{u}_{c}\right|=\text { constant }
$$

where $\mathbf{u}_{c}$ represents the tangential velocity on the cavity surface. The implication of this condition is that the velocity potential at any point $\mathbf{x}$ on the cavity surface can be expressed as

$$
\phi(\mathbf{x})=\phi\left(\mathbf{x}_{d}\right)+\left|\mathbf{u}_{c}\right| s
$$

where the subscript $d$ represents the detachment point of the cavity and $s$ the distance between the detachment point and $\mathbf{x}$ along the cavity surface.

Furthermore, the cavitation number, $\sigma$, can be related to $\mathbf{u}_{c}$ as follows,

$$
\sigma=\frac{P-p_{v}}{\frac{1}{2} \rho U^{2}}=\left(\frac{\left|\mathbf{u}_{c}\right|}{U}\right)^{2}-1,
$$

where $P$ is the pressure of the undisturbed flow and the density of fluid.

For a cavitating flow, we need two more conditions, i.e., the detachment condition and the termination condition of the cavity. These two conditions prescribe where the cavity begins and how it ends. Generally speaking, these conditions cannot be discussed precisely within the context of potential flow since the flow detachment points are strongly affected by viscous effect and the flow around the cavity end is obviously turbulent. For simplicity, we assume that the flow detaches exactly at the leading edge. It is also assumed 
that the flow detaches at the trailing edge for a supercavitating flow.

The termination condition and the related models will be discussed in detailed in the next section.

Finally, for a two-dimensional potential flow in a multi-connected domain, the Kutta condition must be prescribed to determine uniquely the circulation. This condition is prescribed at the end point of cavity for a supercavitating flow.

\section{CAVITY CLOSURE MODELS}

Two different closure models were studied. We briefly describe and comment on these models in the following.

The first one is the simple closure model which, shown in Figure 2, prescribes a simple close cavity on which surface the tangential velocity is constant everywhere, according to Eqs. (5) and (6). This model was employed in [11]. The cavity shape and the tangential speed on the cavity surface are part of the solution to be determined.

This is a purely computational model due to its simplicity in numerical implementation. In fact, from the mathematical point of view, a closed finite body with a constant pressure does not exist in exact potential flow theory. This kind of termination condition is contradictory to the constant pressure condition inside the cavity (or the constant tangential velocity condition on the cavity surface).

The second model is the short plate termination model (or modified Riabouchinsky model). This is a well-developed model proposed by Riabouchinsky [12]. As shown in Figure 3, the cavity ends at a plate normal to the incoming flow. In addition to the cavity shape and the tangential velocity, the height of the short plate is unknown and need be determined as the flow solution is sought.

The short plate model was first employed by Uhlman [16]. Incorporating with this model, he developed a velocity-based nonlinear boundary element scheme to compute supercavitating flow fields.

\section{NUMERICAL DISCRETIZATION}

According to Green's identity, the solution of the

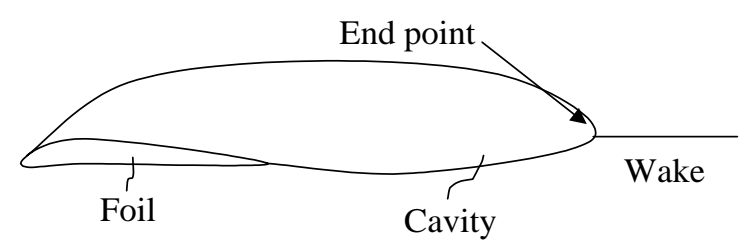

Fig. 2. The constant-pressure model.
Laplace equation, Eq. (1) can be written in terms of normal dipoles and sources distributed on the boundary surfaces. Here, we introduced a dipole distribution on the cavity and wet foil surfaces and a source distribution on the cavity surface. The total potential of the flow may be expressed as

$$
\begin{aligned}
\phi(\boldsymbol{x}) & =\mathbf{U} \cdot \mathbf{x}+\int_{S_{c}} q(\xi) \phi_{S} d S-\int_{S_{b}+S_{c}} \mu(\xi)\left(\mathbf{n} \cdot \nabla \phi_{x}\right) d S \\
& +\int_{S_{w}} \mu_{w}(\xi)\left(\mathbf{n} \cdot \nabla \phi_{x}\right) d S
\end{aligned}
$$

where $q(\xi)$ and $\mu(\xi)$ represent the source and dipole strengths at position $\xi$, respectively, $\phi_{\mathbf{x}}$ is the potential due to a source of unit strength located at $\mathbf{x}$ on the boundary surface, $\mu_{w}$ the dipole strength on the wake surface $S_{w}$, and $\mathbf{n}$ the unit normal directed into the flow at $\xi$.

The distribution of sources on the cavity surface serves as a normal flux generator so as to adjust the form of cavity surface. They vanish pointwise when the cavity surface is exactly prescribed or located, leaving only a dipole distribution on the foil and cavity surfaces.

The numerical discretization has been a standard procedure. The wetted part of the hydrofoil surface and the cavity surface are approximated by an $N$-sided polygon defined by $N_{b}$ and $N_{c}$ vertex points ("nodes") distributed on the surfaces $S_{b}$ and $S_{c}$, respectively. The total number of elements $N$ is the sum of $N_{b}$ and $N_{c}$. Assume that the dipole and source strengths on each element are constant and that the control point is positioned at the center of each element. The discretized form the integral formulation can be formally written as

$$
\phi_{i}=\mathbf{U} \cdot \mathbf{x}_{i}+\sum_{i=1}^{N_{c}} q_{j} \alpha_{i j}-\sum_{k=1}^{N} \mu_{k} \beta_{i k}+\mu_{w} \beta_{i w}
$$

where the subscript $i$ denotes the control point of $i$-th element and the coupling coefficient is defined as follows,

$$
\alpha_{i j}=\frac{1}{2 \pi} \int_{S_{j}} \ln r d S
$$

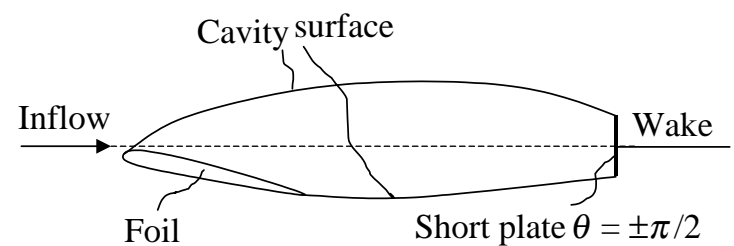

Fig. 3. The modified riabouchinsky model. 


$$
\beta_{i k}=\left\{\begin{array}{lr}
\frac{1}{2 \pi} \int_{S_{j}} \frac{\partial \ln r}{\partial n_{k}} d S, & i \neq k, \\
\frac{1}{2}, & i=k
\end{array}\right.
$$

where $r$ is the distance between points $\xi$ and $\mathbf{x}$.

To obtain the solution, one must solve together the unknown cavity shape and discretized source and dipole strengths in the equation system (9). Therefore, the singularity strengths and locations on the cavity surface must be updated iteratively. A nonlinear procedure has been developed to cope with this kind of solution. The detailed description of the iterative procedure can be found in [3]. For completeness, it is briefly stated in the following.

(1) Prescribe the cavity length and an initial shape of cavity surface.

(2) Determine the dipole and source strengths by solving the equation system of Eq. (9) on the basis of the prescribed cavity boundary.

(3) The shape of cavity surface is then updated based on the source distribution obtained in the last step.

(4) Repeat steps (2) and (3) till reasonable convergence is achieve.

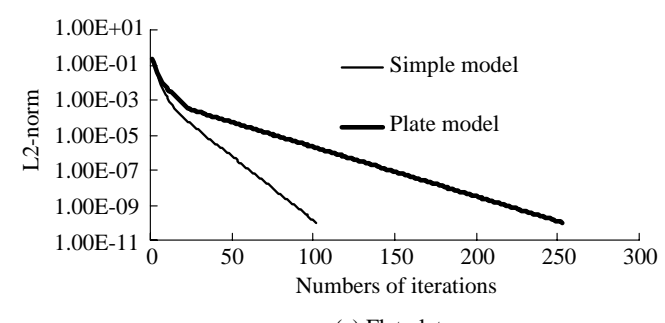

(a) Flat plate.

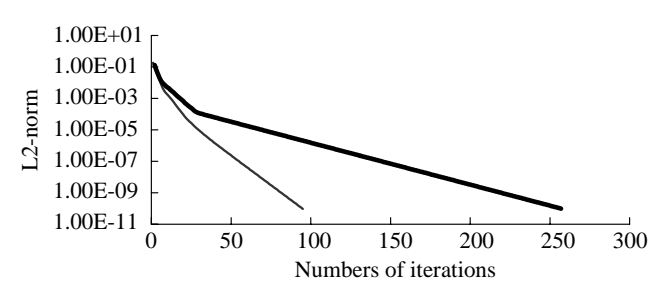

(b) NACA16004

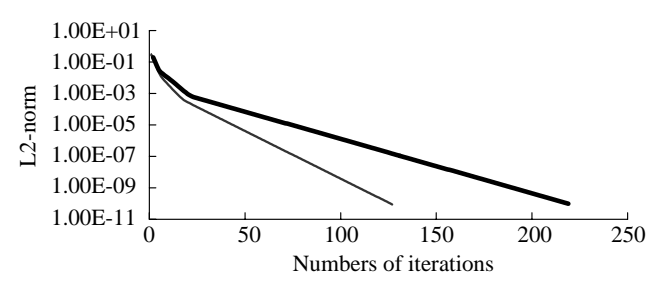

(c) Eppler plate.

Fig. 4. Convergence history for different hydrofoils at $\alpha=4^{\circ}$ and $\ell / c=\mathbf{1 . 2}$.

\section{SOME TEST CASES AND DISCUSSIONS}

For test purposes, a series of computations have been conducted for three different hydrofoil sections. They are flat plate, NACA16004 section, and Eppler's new section. The flat-plate hydrofoil is the most typical case in literature because its analytical data are available. The NACA16004 section represents a "traditional" hydrofoil section. In contrast, the Eppler's new section represents a hydrofoil section of new generation designed specifically for cavitating flow conditions. For each hydrofoil, we varied the cavity-length-to-chord ratio $(\ell / c=1.2,1.4,1.6,1.8,2.0)$ and the angle of attack $\left(\alpha=2^{\circ}, 4^{\circ}, 6^{\circ}, 8^{\circ}\right)$.

In addition to compare effects of different closure models on the flow development, we employed the same mesh in the chordwise direction for the each flow setup condition. For the short plate model, two additional elements were implemented on the plate to model plate effects. Some important effects we have observed are discussed in the following.

We first examine the cavity shape. Some of typical convergent computational results for the simple and short-plate models are shown in Figures 4-7. Figures 4 and 5 show the convergence history $\left(L_{2}\right.$-norm of difference between two subsequent iterative solutions) and the cavity shape, respectively, for different hydrofoil sections at a small flow angle of attack, $\alpha=4^{\circ}$, and a short cavity length, $\ell / c$. On the other hand, Figures 6 and 7 show results at a bigger angle of attack, $\alpha=8^{\circ}$, and a longer cavity length $\ell / c$.

For each case, regardless of the incidence angles
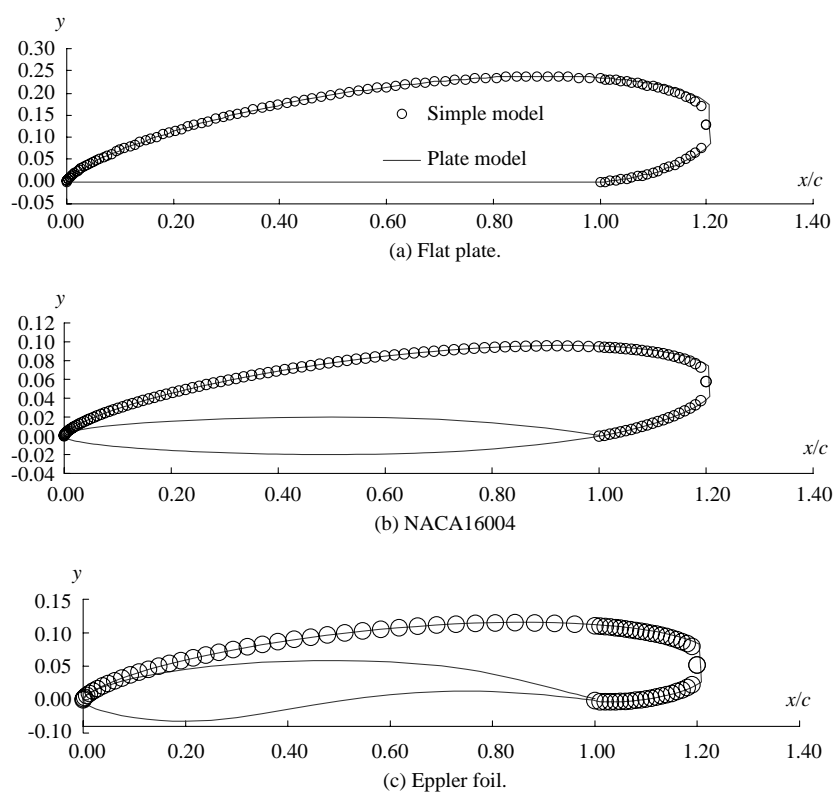

Fig. 5. Cavity shapes for different hydrofoils at $\alpha=4^{\circ}$ and $\ell / c=1.2$. 
of flow and cavity lengths, we find that the cavity shape curves closely coincide to each other, except at the rear cavity region where the closure models are applied. This seems to indicates that different closure models do not significantly alter the cavity shape.

It is interesting to note that, as pointed out in the previous section, the simple closure model contradicts to the potential flow theory and, therefore, cannot be a real physical model. However, this fact does not create a significant deviation of cavity shape. Therefore, as far as computations and engineering applications are concerned, this seems to be a feasible model. In fact, we find that the simple closure model is a good one in terms of not only its engineering applicability but also its robust convergence property that will be discussed later.

In addition, since the cavity shapes computed from different closure models do not differ significantly, we may conclude immediately that the cavity volumes vary insignificantly.

The cavitation numbers we obtained by computations with three different closure models do not vary significantly. Furthermore, general observations throughout our test cases also show that the static pressure distributions on the foil surfaces agree with one another very well. This implies that at a specified set of flow incidence and cavity length, the lifts due to different closure models do not have observable deviation from one another.

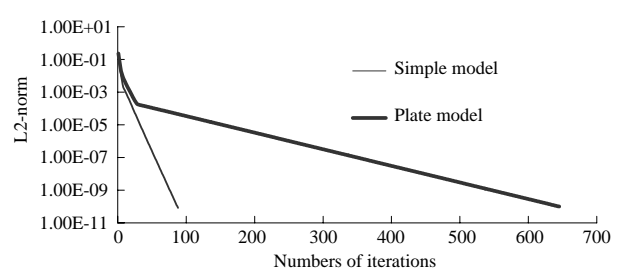

(a) Flat plate
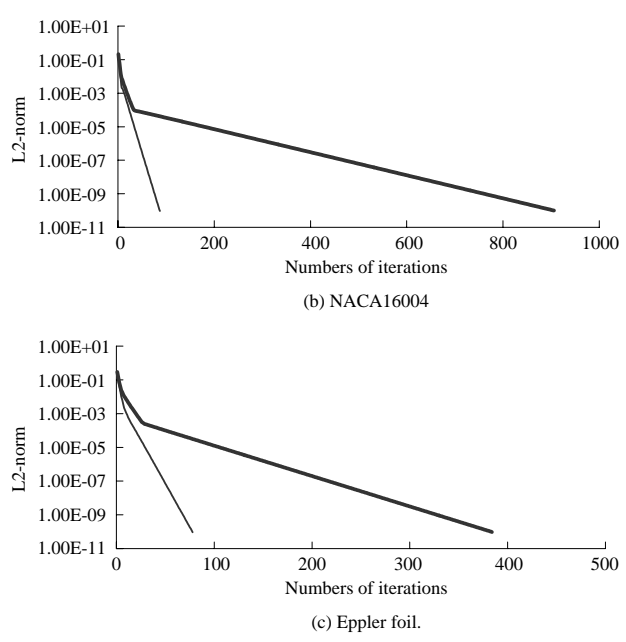

Fig. 6. Convergence history for different hydrofoils at $\alpha=8^{\circ}$ and $\ell / c=\mathbf{2 . 0}$.
Observing these computational results, we may conclude that, as far as macroscopic properties of cavity are concerned, cavity closure models have only minor effects on the development of flows of two-dimensional supercavitating hydrofoils.

Nevertheless, the convergence history of iterations shows an interesting phenomenon for different closure models. As shown in Figures 4 and 6, it appears that computations with the simple closure model have a much better and stable convergence history. In contrast, those employing the short-plate model usually require much more number of iterations to converge. What is worse is that the iterations sometimes lead to divergence.

Figure 8 shows the convergence history at $a=2^{\circ}$ and $\ell / c=1.4$. Iterated with the same initial guess, the computations that incorporated with the simple model were fully convergent within 100 iterations whereas those with the short-plate model could not achieve a reasonable convergence. In fact, we found that for all three hydrofoils the computations with the short-plate model were usually divergent for a long cavity if the angle of attack was small. It seems that the short-plate model is somewhat unstable for iterations of the present potential-based scheme. Nevertheless, it appears that this model does not show such divergent iterations in the velocity-based scheme developed by Uhlman [16] in which the unknown is the surface vorticity rather than velocity potential. The reason why the employment of the modified Riabouchinsky model in the present potential-based boundary element method leads to numerical instability in some cases is not clear. A possible cause
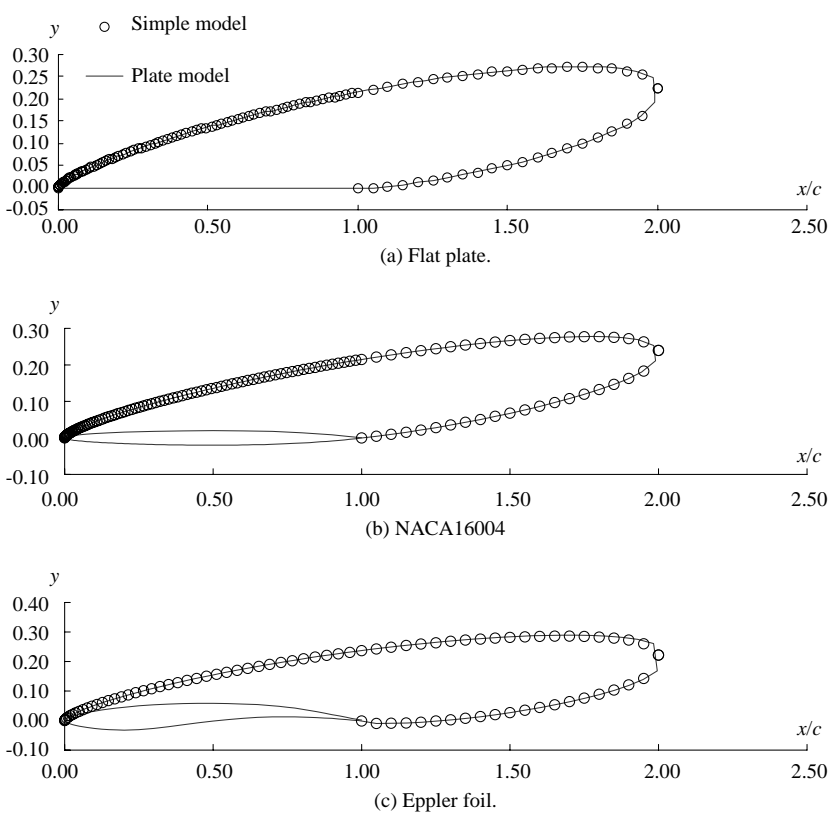

Fig. 7. Cavity shapes for different hydrofoils at $\alpha=\mathbf{8}^{\circ}$ and $\ell / c=\mathbf{2 . 0}$. 
may be due to sharp edges of streamline near the ends of the short plate, which is detrimental in finding the tangential velocity on the cavity surface by taking first derivatives of the velocity potential. This is especially true for a long bubble with a small angle of attack. The velocity-based scheme avoids this dilemma because it employs surface vorticity as unknown from which the velocity distribution can be immediately obtained without taking partial derivatives.

Nevertheless, as the angle of attack increases, the flat-plate model leads to convergence. Figure 9 shows such an example for which the angle of attack is and the cavity length ratio is 1.4. However, it is evident that the

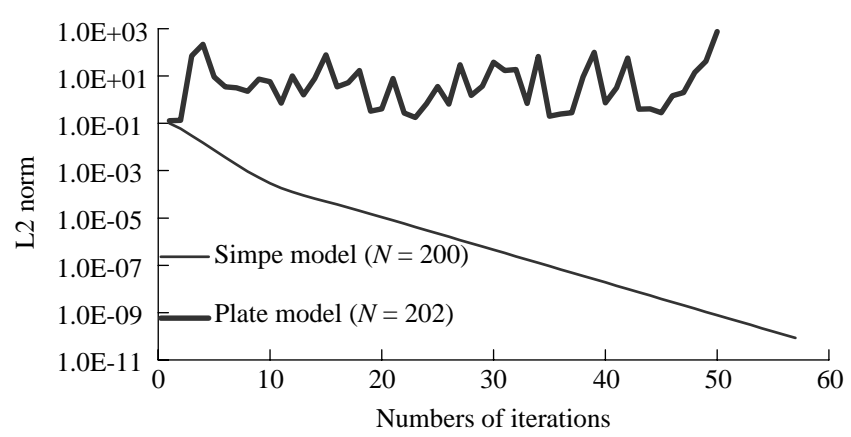

Fig. 8. Convergence history for flat-plate hydrofoils at $\alpha=2^{\circ}$ and $\ell / c=\mathbf{1 . 4}$.

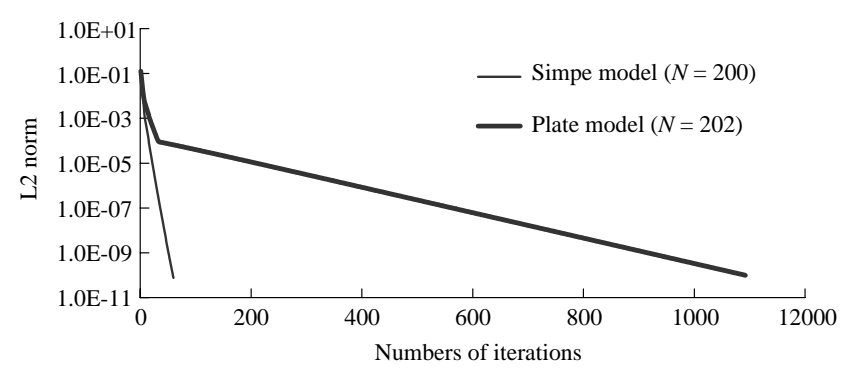

Fig. 9. Convergence history for NACA16004 hydrofoils at $\alpha=4^{\circ}$ and $\ell / c=\mathbf{1 . 4}$.

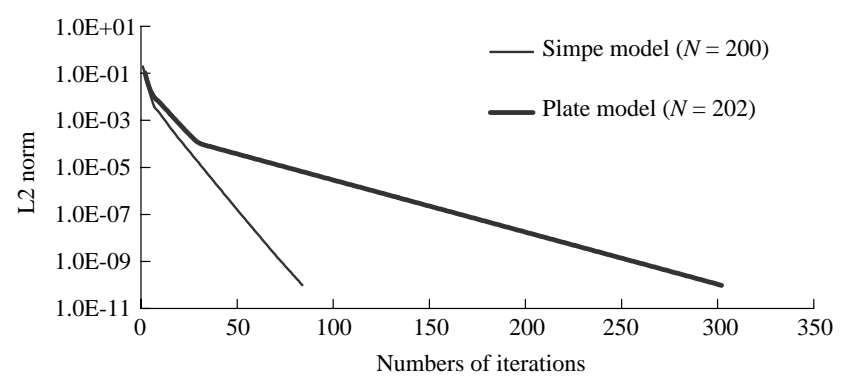

Fig. 10. Convergence history for NACA16004 hydrofoils at $\alpha=6^{\circ}$ and $\ell / c=1.4$. computation with the flat-plate model took much longer time to converge, compared to that with the simple closure model. The latter converged within 100 iterations, whereas the former did not fully converge before 1000 iterations.

For an even larger angle of attack, the convergence history became better for the flat-plate model, as shown in Figure 10. Nevertheless, the simple closure model is still a better scheme to be employed in computations because of its better convergence rate. Similar behaviors of convergence were observed for other hydrofoils. It seems that these trends are universal.

\section{CONCLUSION}

Our study shows that cavity closure models do not have strong effect on the development of the cavity and engineering integrated properties of interests in supercavitating flows. Even though the reentrant jet model is known to its better approximation to real supercavitating flows, the computation results show that they do not deviate significantly from those by simpler closure models.

What should be more cautious in using these models is the convergence problem in computations. Different models incur different convergence processes and in some cases result in divergence during iterations. Therefore, as far as engineering applications are concerned, the simple closure model should be a better choice because of its easiest implementation and numerical robustness in iterations.

\section{ACKNOWLEDGEMENTS}

This work was supported by the National Science Council, Republic of China, under the grant NSC912815-C-019-013-E. The authors would like to express their thanks to this support.

\section{REFERENCES}

1. Acosta, A.J., A Note on Partial Cavitation of Flat Plate Hydrofoils (Hydrodynamics Laboratory Report E19.9), California Institute of Technology, Pasadena, CA (1955).

2. Brennen, C.E., Cavitation and Bubble Dynamics, Oxford University Press, NewYork (1955).

3. Chen, J.-H. and Weng, Y.-C., "Analysis of Flow Past a Two-Dimensional Supercavitating Hydro-Foil Using a Simple Closure Model," P. Natl. Sci. Counc., ROC(A), Vol. 23, pp. 411-418 (1999).

4. Dang, J. and Kuiper, G., "Re-entrant Jet Modeling of Partial Cavity Flow on Two-Dimensional Hydrofoils," Proceedings of the Third Symposium on Cavitation, Grenoble, France (1998). 
5. Efros, D.A., "Hydrodynamical Theory of Two-Dimensional Flow with Cavitation," Dokl. Akad. Nauk SSSR, Vol. 51, pp. 267-270 (1946).

6. Furuya, O. and Acosta, A.J., "A Note on the Calculation of Supercavitating Hydrofoils with Rounded Noses," J. Fluid Eng-T ASME, Vol. 95, pp. 222-228 (1973).

7. Kinnas, S.A. and Fine, N.E., "A Numerical Nonlinear Analysis of the Flow Around Two and Three-Dimensional Partially Cavitating Hydrofoils," J. Fluid Mech., Vol. 254, pp. 151-181 (1993).

8. Kirchhoff, G., "Zur Theorie freier Flussigkeitsstrahlen," Z. Reine Angew. Math., Vol. 70, pp. 289-298 (1869).

9. Kreisel, G., Cavitation with Finite Cavitation Numbers (Technical Report No. R1/H/36), Admiralty Research Laboratory, Teddington, UK (1946).

10. Krishnaswamy, P., Andersen, P., and Kinnas, S.A., "Reentrant Jet Modeling for Partially Cavitating Two-Dimensional Hydrofoils," Proceedings of the 4th International Symposium on Cavitation, CAV2001, Pasadena, CA (2001).

11. Lee, C.S., Kim, Y.G., and Lee, J.T., “A Potential-Based Panel Method for the Analysis of a Two-Dimensional Super- or Partially-Cavitating Hydrofoil," J. Ship Res., Vol. 36, pp. 168-181 (1992).

12. Riabouchinsky, D., "On Steady Fluid Motion with a Free
Surface," P. Lond. Math. Soc., Vol. 19, pp. 206-215 (1920).

13. Tulin, M.P., Steady Two-Dimensional Cavity Flows About Slender Bodies (Report No. 834), David Taylor Model Basin, Washington, DC (1953).

14. Tulin, M.P., "Supercavitating Flows - Small Perturbation Theory," J. Ship Res., Vol. 7, pp. 16-37 (1964).

15. Uhlman, J.S., "The Surface Singularity Method Applied to Partially Cavitating Hydrofoils," J. Ship Res., Vol. 31, pp. 107-124 (1987).

16. Uhlman, J.S., “The Surface Singularity or Boundary Integral Method Applied to Supercavitating Hydrofoils," J. Ship Res., Vol. 33, pp. 16-20 (1989).

17. Vaz, G., Bosschers, J., and Falcão de Campos, J.A.C., "Analysis of Two Boundary Element Methods for Cavitation Modeling," Proceeding of the 5th International Symposium on Cavitation, CAV2003, Osaka, Japan (2003).

18. Wu, T.Y., "A Free Streamline Theory for Two-Dimensional Fully Cavitated Hydrofoils," J. Math. Phys., Vol. 35, pp. 236-265 (1956).

19. Wu, T.Y., "A Wake Model for Free Streamline Flow Theory, Part 1. Fully and Partially Developed Wake Flows and Cavity Flows past an Oblique Flat Plate," $J$. Fluid Mech., Vol. 13, pp. 161-181 (1962). 